\title{
Isolated Bilateral Fourth Cranial Nerve Palsies as the Presenting Sign of Hydrocephalus
}

\author{
Dimosthenis Mantopoulos ${ }^{a, c}$ David G. Hunter ${ }^{b, c}$ \\ Dean M. Cestaria, c \\ aNeuro-Ophthalmology Service, Department of Ophthalmology, Massachusetts \\ Eye and Ear Infirmary, ${ }^{b}$ Children's Hospital Boston, and 'Department of \\ Ophthalmology, Harvard Medical School, Boston, Mass., USA
}

\section{Key Words}

Strabismus $\cdot$ Trochlear $\cdot$ Headache $\cdot$ Sylvian aqueduct

\begin{abstract}
Midbrain lesions leading to bilateral fourth nerve palsies are typically accompanied by other brainstem symptomatology. Here we report a case of a 29-year-old man with hydrocephalus and significant third ventricle dilation applying pressure on the dorsal midbrain and having as the only manifestation isolated, bilateral fourth cranial nerve palsies. This finding, reported here for the first time, could be attributed to a partially working ventriculoperitoneal shunt previously placed in this patient, which was able to sporadically relieve the increases of the intraventricular pressure on the midbrain that would normally lead to other manifestations.
\end{abstract}

\section{Introduction}

Hydrocephalus results from an abnormal accumulation of cerebrospinal fluid in the ventricles of the brain and the clinical presentation varies with chronicity. Acute dilatation of the ventricular system is more likely to manifest with nonspecific signs and symptoms of increased intracranial pressure including headache and tinnitus. By contrast, chronic dilatation may have a more insidious onset, often presenting with dementia, unsteady gait or incontinence. Focal neurologic deficits such as an abducens nerve palsy or impaired vertical gaze may also develop due to compression of the quadrigeminal plate, where the neural centers coordinating vertical eye movement are located. Acutely, patients are often successfully treated with ventriculoperitoneal shunts (VPS) but these shunts have a relatively high failure rate over time. We report a case of a 
patient who presented with bilateral 4 th nerve palsies as the only manifestation of $3 \mathrm{rd}$ ventricular hydrocephalus that resulted from a malfunctioning VPS.

\section{Case Report}

A 29-year-old man was referred to a tertiary care neuro-ophthalmology department complaining of binocular vertical diplopia worse in downgaze, especially when reading. He also noted daily frontal headaches for the past 2 weeks. His past medical history was significant for congenital biliary atresia treated with a portomesenteric shunt, followed by liver transplantation when the shunt failed 11 years later. He developed a subarachnoid hemorrhage within the same year, which prompted a diagnostic evaluation that revealed multiple intracranial arterial aneurysms. Subsequent hydrocephalus required ventriculostomy of the 3rd ventricle followed by the placement and revision of a VPS multiple times over the next decade. Additionally, he had a history of systemic hypertension, migraine headaches and congenital color blindness. His medications included cyclosporine, azathioprine, sulfamethoxazole and trimethoprim, and atenolol.

The initial neuro-ophthalmic examination revealed an alert and cooperative patient with a best corrected visual acuity of $20 / 25-2$ OD and $20 / 20-2$ OS. He was able to correctly identify $2 / 8$ Ishihara color plates OU. Pupils were equal, round and reactive without a relative afferent pupillary defect and no light-near dissociation. Upgaze was intact and there was no retraction nystagmus. The senosorimotor evaluation demonstrated a right hypertropia of 5 prism diopters (PD) in left gaze and a left hypertropia of 6 PD in right gaze, as well as a V-pattern esotropia. Double Maddox rod testing showed 15 degrees of excyclotorsion and ductions were full. These findings in the setting of new-onset headaches prompted neuroimaging ( $\underline{\text { fig. }}$ 1a, b). Sagittal and axial MRI sequences demonstrated large $3 \mathrm{rd}$ and 4 th ventricular dilation that appeared to exert pressure on the dorsal midbrain. The neurosurgical consultation confirmed a dysfunctional VPS. Subsequently, the patient underwent multiple attempts at revising the shunt and ultimately had the shunt removed and a new ventriculostomy was performed with resolution of the hydrocephalus as well as the left 4th cranial nerve palsy (fig. 1c, d).

\section{Discussion}

Bilateral 4th cranial nerve palsies are characterized by an ipsilateral hypertropia that manifests on contralateral gaze. They are typically associated with 10-15 degrees of excyclotorsion as well as a V-pattern esotropia. The most common etiology is significant head trauma, although any lesion involving the superior medullary velum, the anatomical location where the two trochlear nerves decussate, can result in this finding. Skew deviation is the main differential diagnosis of 4 th nerve palsies and they are associated with a concomitant hypertropia and a torsional defect that is typically less than 5 degrees [1].

Ciardella et al. [2] reported three patients with bilateral 4th nerve palsies in the setting of nonneoplastic hydrocephalus. Each of their patients had concomitant dorsal midbrain findings including limitation of upgaze, light-near dissociation and convergenceretraction nystagmus, suggesting there was downward pressure on the midbrain from an enlarged 3rd ventricle. To our knowledge, this is the first case report of a patient with isolated bilateral 4 th nerve palsies from an enlarged 3rd ventricle resulting from a malfunctioning VPS. It is possible that our patient did not manifest midbrain findings because his VPS was partially functioning, thereby allowing only a pressure elevation great enough to cause trochlear nerve dysfunction and not sufficient enough to cause midbrain dysfunction. 
Our patient had two uncommon diagnoses that include congenital biliary cirrhosis and multiple intracranial arterial aneurysms, one of which ruptured during childhood. These findings can be seen in patients with Alagille syndrome, an autosomal dominantly inherited disease implicating the genes JAG1 or notch $2[3,4]$. However, additional features of this syndrome, including heart, renal, skeleton and facial features, were absent, and there were no ophthalmologic manifestations, such as posterior embryotoxon, pupil or optic nerve anomalies nor retinitis pigmentosa. He did have a history of systemic arterial hypertension, though this only occurred after the introduction of high-dose prednisone following the liver transplantation.

To our knowledge, this is the first case report of isolated bilateral 4th cranial nerve palsies as the only manifestation of significant dilation of the Sylvian aqueduct and 3rd ventricle. This finding can indicate the failure of a previously functioning VPS, as in our patient, and we believe it should prompt neuroimaging to exclude hydrocephalus as a causative etiology.

\section{Disclosure Statement}

The authors do not have a conflict of interest. 

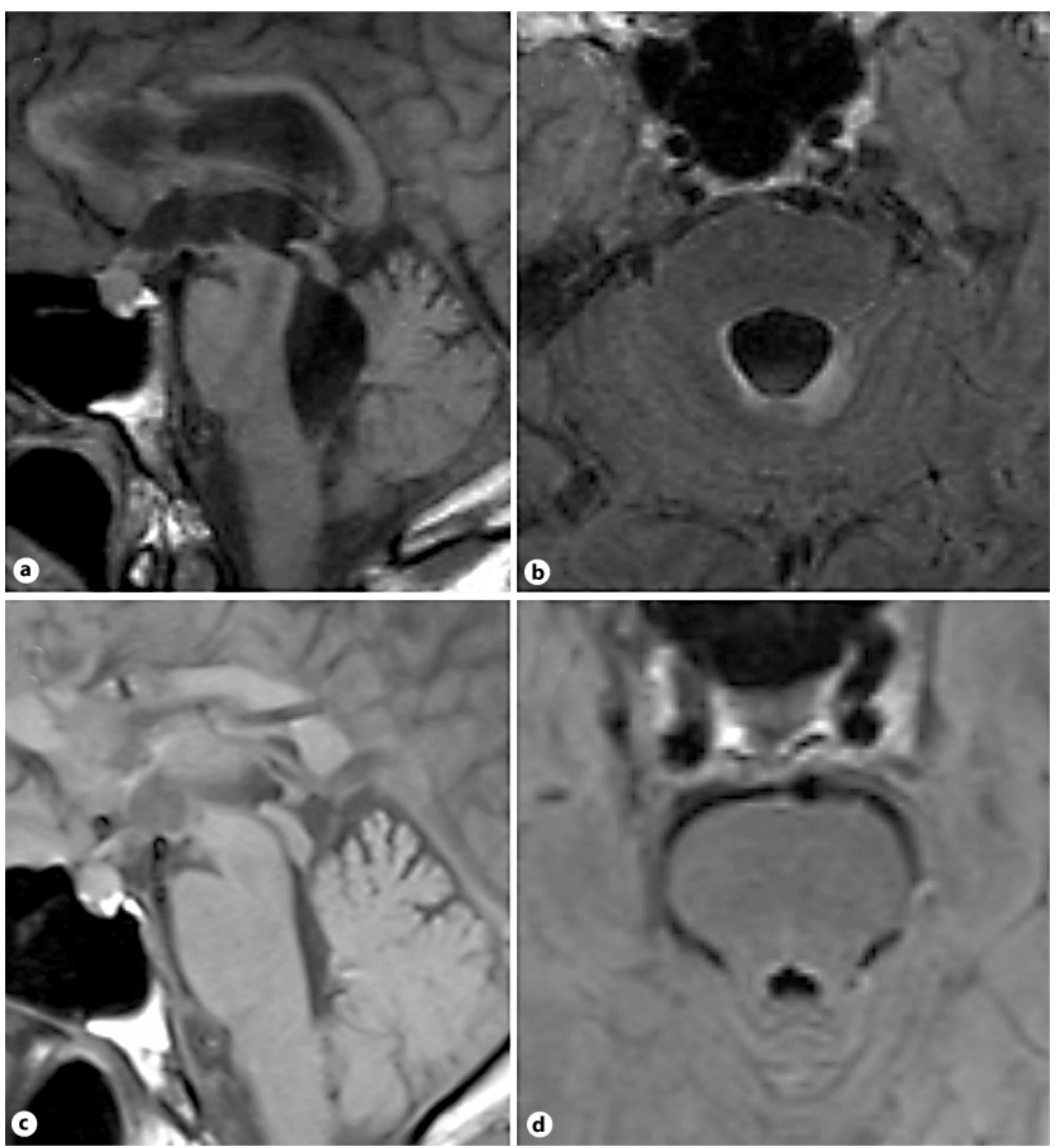

Fig. 1. Brain MRI study. a, b Magnified views of 3rd and 4th ventricular dilation at presentation. a Sagittal T1. b Axial FLAIR sequence. c, d Magnified views after ventriculostomy. c Sagittal T1. d Axial T1 sequence.

\section{References}

1 Brodsky MC, Donahue SP, Vaphiades M, Brandt T: Skew deviation revisited. Surv Ophthalmol 2006;51:105128.

2 Ciardella AP, Fisher YL, Carvalho C, Slakter JS, Bryan RG, Sorenson JA, et al: Endoscopic vitreoretinal surgery for complicated proliferative diabetic retinopathy. Retina 2001;21:20-27.

3 Li L, Krantz ID, Deng Y, Genin A, Banta AB, Collins CC, et al: Alagille syndrome is caused by mutations in human Jagged1, which encodes a ligand for Notch1. Nat Genet 1997;16:243-251.

4 McDaniell R, Warthen DM, Sanchez-Lara PA, Pai A, Krantz ID, Piccoli DA, et al: NOTCH2 mutations cause Alagille syndrome, a heterogeneous disorder of the notch signaling pathway. Am J Hum Genet 2006;79:169173. PMCID: 1474136. 